\title{
Quality measures for the management of hydrocephalus: concepts, simulations, and preliminary field-testing
}

\author{
Clinical article
}

\author{
Spencer E. Barton, ${ }^{1}$ Jeffrey W. Campbell, M.D., M.S., ${ }^{2,3}$ and Joseph H. Piatt JR., M.D. ${ }^{2,3}$ \\ ${ }^{1}$ Carnegie Mellon University, Pittsburgh; ${ }^{3}$ Departments of Neurological Surgery and Pediatrics, Thomas \\ Jefferson University, Philadelphia, Pennsylvania; and ${ }^{2}$ Division of Neurosurgery, Alfred I. duPont Hospital \\ for Children, Wilmington, Delaware
}

\begin{abstract}
Object. The authors define and examine the properties of 2 new, practice-based quality measures for the management of hydrocephalus.

Methods. The Surgical Activity Rate (SAR) is defined as the number of definitive operations for the treatment of hydrocephalus performed in a neurosurgical practice over the course of a year, divided by the number of patients with hydrocephalus seen in follow-up during that year. The Revision Quotient (RQ) is defined as the number of definitive revision operations performed in a neurosurgical practice in the course of a year, divided by the number of definitive initial operations during that year for patients with newly diagnosed hydrocephalus. Using published actuarial shunt survival data, the authors conducted Monte Carlo simulations of a pediatric neurosurgical practice to illustrate the properties and interpretations of the SAR and RQ. They used data from the Kids' Inpatient Database (KID) for 2009 to calculate RQs for hospitals accounting for more than 10 admissions coded for initial CSF shunt insertions.

Results. During the initial growth phase of a simulated neurosurgical practice, the SAR approached its steadystate value much earlier than the RQ. Both measures were sensitive to doubling or halving of monthly failure rates. In the $2009 \mathrm{KID}, 117$ hospitals reported more than 10 initial shunt insertions. The weighted mean ( \pm standard deviation) RQ for these hospitals was $1.79 \pm 0.69$. Among hospitals performing 50 or more initial shunt insertions, the RQ ranged between 0.71 and 3.65 .

Conclusions. The SAR and RQ have attractive qualitative features as practice-based quality measures. The RQ, at least, exhibits clinically meaningful interhospital variation as applied to CSF shunt surgery. The SAR and RQ merit prospective field-testing as measures of quality in the management of childhood hydrocephalus. (http://thejns.org/doi/abs/10.3171/2013.1.PEDS12362)
\end{abstract}

\section{KEY WORdS • endoscopic third ventriculostomy • hydrocephalus • quality measure $\quad$ shunt}

$\mathrm{F}$ OR the past 6 decades the CSF shunt has been the workhorse of the treatment of hydrocephalus. Compared with what came before, CSF shunting is a highly effective therapy, but its high complication rate has made it a burdensome therapy as well. In the past 2 decades, the burden of shunt surgery for selected patients has been lifted via ETV, but the most skilled hands exercising the best judgment seldom achieve initial success rates in excess of $60 \% .{ }^{11}$ In even the most favorable scenario, patients in whom ETV fails are often subjected to repeat ETV, and most eventually require life-long shunt

Abbreviations used in this paper: ETV = endoscopic third ventriculostomy; HOQ = Hydrocephalus Outcome Questionnaire; KID = Kids' Inpatient Database; PHIS = Pediatric Health Information System; SAR = Surgical Activity Rate; RQ = Revision Quotient. maintenance. Thus children with newly diagnosed hydrocephalus and their families face futures of repeated hospitalizations at unpredictable intervals. Minimization of these disruptive events is clearly a desirable goal.

The burden of the surgical treatment of hydrocephalus can be analyzed from several perspectives: the operation, the patient, and the neurosurgeon or neurosurgical practice. Many published actuarial data have described the failure rates of operations-both CSF shunt surgery and ETV. ${ }^{2-6,8,14,16,17}$ Such data are relatively easy to obtain and analyze for the purposes of clinical research but are difficult for patients and families to relate to their own lives. Data on the longitudinal experiences of individual patients might be of much greater interest, but because of the long periods of observation required, they are very difficult to collect. Indeed they are very scarce in the lit- 


\section{Quality measures for the management of hydrocephalus}

erature. Piatt and Cosgriff ${ }^{15}$ performed a digital simulation of the 10-year experiences of individuals with newly diagnosed hydrocephalus by using published shunt survival data. This exercise produced estimates of expected numbers of hospitalizations over the simulated study period with $95 \%$ and $99 \%$ confidence intervals. Simulated statistics about hospitalizations over time can be useful as background information for counseling, but they are not actionable: Practitioners cannot use them for quality improvement. Neither can patients and families use them to choose among practitioners.

We propose 2 neurosurgical practice-based quality measures that fulfill these purposes. The Surgical Activity Rate (SAR) is defined as the number of definitive operations for the treatment of hydrocephalus performed in a neurosurgical practice over the course of a year, divided by the number of patients with hydrocephalus seen in follow-up during that year. Only operations intended to stabilize the patient for discharge from the hospital are counted; external ventricular drain placements, shunt externalizations, and shunt removals without replacement are not considered. The Revision Quotient (RQ) is defined as the number of definitive revision operations performed in a neurosurgical practice in the course of a year-either shunt revisions or ETV revisions-divided by the number of definitive initial operations during that year for patients with newly diagnosed hydrocephalus. What follows is a series of digital simulations to serve as an introduction to the interpretation of these measures and preliminary calculations of one of these measures, the RQ, from a nationwide administrative data set.

\section{Methods}

The simulated neurosurgical practices were limited to patients younger than 18 years. With every year of the simulation, a constant number of new virtual patients entered each practice, with ages randomly determined from a probability distribution modeled on the ages of patients undergoing initial CSF shunt insertion in the Shunt Design Trial. ${ }^{3}$ Individual patients were aged on a month-wise basis and left the practice at the end of their 216 th month (18th year) of life. The simulated practices thus grew steadily in the number of patients under care for the first 18 years and then stabilized. The simulations were run for 100 years. Although patients under care, annual operations, SARs, and RQs seemed to stabilize by the 19th year, the last 60 years of each simulation were defined as the practice's steady state. Simulations were conducted for small and large practices, with 20 and 100 new patients per year, respectively.

The surgical experiences of each virtual patient were simulated as reported previously. ${ }^{15}$ Briefly, actuarial shunt survival data were developed in life-table format from a previously published institutional case series with exponential extrapolation to reach 216 months. ${ }^{14}$ Each new patient's initial shunt insertion was counted in the year of the patient's entry into the simulation. For each patient for each subsequent month, a random number between 0 and 1 was generated. If the random number was greater than the life-table probability of failure of the shunt in that month, the patient and the patient's shunt went on to the next month of the life table. If the random number was less than the probability of shunt failure, one additional operation was counted for that year of the simulation, and the patient's shunt returned to the 1st month of the life table. After the 216th month the patient transitioned out of the simulated practice. The output of the simulation was the count of operations performed in each year.

Code for the simulation was written in Python (version 2.7, Python Software Foundation), an open-source, high-level programming language.

The actuarial data developed from the institutional data set is referenced as Practice A. Shunts that require revision after a short time interval from the preceding operation are believed to be at higher risk for failure than new shunts or shunts requiring revision after a long interval. ${ }^{14,18}$ To enhance the realism of the model, a second set of simulations were performed with a life table modified for shunts revised after less than 6 months from the preceding operation. For these patients who experienced early failures, the monthly probability of failure of the revised shunt was augmented by a multiplicative factor of 1.5 for the next 6 months. These modified actuarial data are referenced as Practice B. To illustrate the effects on quality measures of markedly better and markedly worse shunt survival, Practices C and D were developed from Practice A with twice and half the monthly risks of failure, respectively.

To determine whether the RQ varies to a meaningful degree among real clinical practices, the Kids' Inpatient Database (KID) was queried. The KID is a compilation of de-identified discharge data from a stratified sample of all hospital discharges of patients in the pediatric age range from community nonrehabilitation hospitals in the US. The database is prepared every 3 years by the Healthcare Utilization Project of the Agency for Healthcare Research and Quality in cooperation with participating states. Each KID includes roughly $10 \%$ of all discharges of uncomplicated in-hospital live births and roughly $80 \%$ of discharges of complicated births and other pediatric cases from each sampled hospital. In the present study we used the 2009 KID, which includes children from birth through 20 years of age. Hospital admissions featuring ICD-9-CM diagnostic codes for hydrocephalus $(331.3,331.4,742.3$, and 741.0x) and procedural codes for CSF shunt insertion $(02.31,02.32,02.33,02.34,02.35,02.39)$ or revision $(02.41,02.42,54.95)$ composed the study group. As there is no procedural code for revision ETV, analysis was limited to CSF shunt surgery. Admission files in the KID include a field for a hospital code. Hospitals with more than 10 admissions for CSF shunt insertion were identified, and RQs were calculated for these hospitals. As the KID is limited to inpatient events, the total number of patients under care at a particular hospital cannot be ascertained from it. Neither can SARs be calculated.

The Nemours Delaware Institutional Review Board judged this investigation to be exempt from oversight.

\section{Results}

The growth phase of a virtual practice based on the 
life table for Practice A accruing 100 patients per year is presented in Fig. 1. The evolution of the SAR and RQ during the growth phase of this practice is presented in Fig. 2. The RQ did not reach its steady-state value until the 18 th year of the simulation, but the SAR was very close to its steady-state value by the 12th year.

Steady-state SARs and RQs for various annual patient accrual rates are presented in Table 1 for simulations of Practices A, B, C, and D. In the steady-state phase of the simulations, total number of patients under care, annual operations, SARs, and RQs were all normally distributed (data not shown).

An issue of practical importance for a quality measure is how much data collection is required to obtain a reasonable estimate of its true value. Tables 2 and 3 depict the estimated number of years of observation required to distinguish the SARs and RQs, respectively, of the simulated practices. (These estimates are based on a priori knowledge of the standard deviations of quality measures derived from the simulations presented in Table 1. In actual field use standard deviations might be estimated from historical data or pilot data.) As constructed for this exercise, Practices A and B are essentially indistinguishable under the SAR and RQ metrics, whereas Practices $\mathrm{C}$ and $\mathrm{D}$ are readily distinguishable from each other and from Practices A and B.

In the 2009 KID 117 hospitals reported more than 10 admissions for the insertion of new CSF shunts. The RQs for these hospitals are presented in Fig. 3. The weighted mean RQ for these hospitals was $1.79 \pm 0.69$. Among hospitals reporting more than 50 new shunt insertions per year, the RQ varied between 0.71 and 3.65.

\section{Discussion}

The SAR and RQ have attractive features as qual-

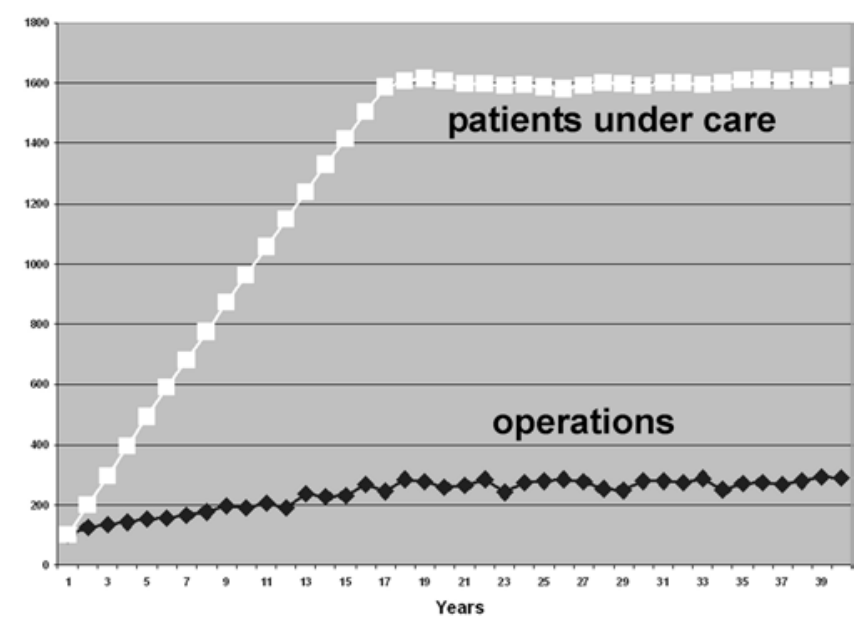

FIG. 1. Graph showing growth of a simulated pediatric neurosurgical practice. In this practice 100 new patients undergo CSF shunt insertion every year. The ages of patients at entry into the simulation are modeled on the age distribution of patients in the Shunt Design Trial. Patients leave the simulation at their 18th birthday. Total patients under care (white squares) and total annual operations (black diamonds) increase steadily through the 18th year and then stabilize with a relatively small year-to-year fluctuation. The y axis indicates number of patients.

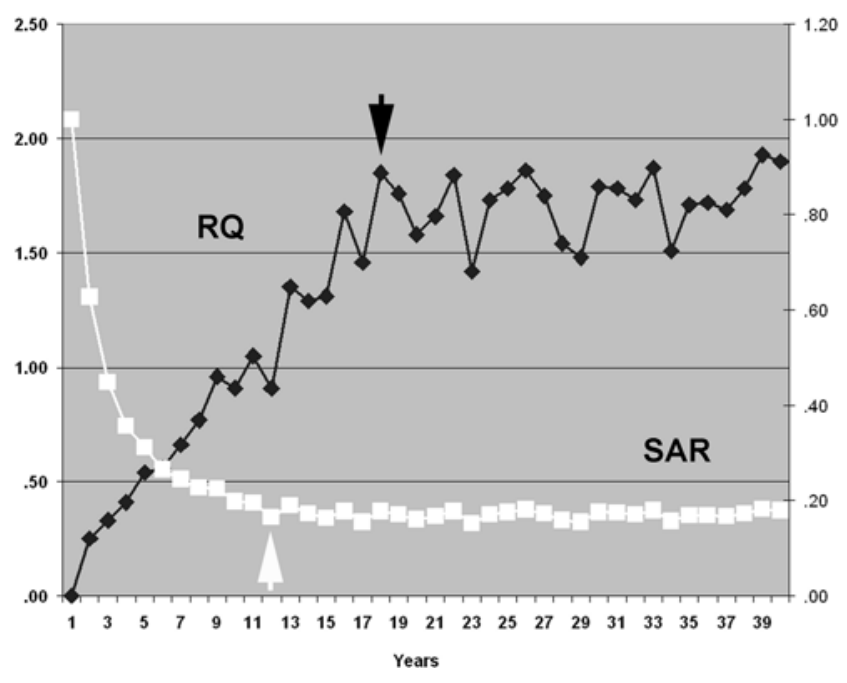

FIG. 2. Graph showing evolution of the SAR (white squares) and $R Q$ (black diamonds) for the simulated pediatric neurosurgical practice depicted in Fig. 1. Note that the SAR reaches its steady-state value by the 12th year (white arrow), while the $R Q$ does not reach its steady-state value until the 18th year (black arrow). Left y axis represents $R Q$; right y axis, SAR.

ity measures in the management of hydrocephalus. Both measures relate directly to events of serious concern to patients, families, and surgeons. The data required for calculating both measures are relatively easy to collect. Our preliminary work indicates that clinically meaningful differences exist among hospitals.

The most important outcome in the management of hydrocephalus is, by definition, quality of life. Measuring the quality of life among children with hydrocephalus has been reviewed. ${ }^{7}$ An intuitive approach to assessing quality of life is the measurement of functional capacity or its inverse, neurological disability, but as a measure of practice-based quality of care, functional capacity is impractical. Quantitative analysis of functional capacity in even a single individual is a labor-intensive process. Among children with hydrocephalus, it is determined

TABLE 1: Steady-State SARs and RQs*

\begin{tabular}{|c|c|c|c|}
\hline Practice & $\begin{array}{c}\text { Annual Patient } \\
\text { Accrual (no.) }\end{array}$ & SAR & $\mathrm{RQ}$ \\
\hline \multicolumn{4}{|l|}{$A$} \\
\hline small & 20 & $0.165 \pm 0.019$ & $1.633 \pm 0.300$ \\
\hline large & 100 & $0.168 \pm 0.009$ & $1.698 \pm 0.140$ \\
\hline \multicolumn{4}{|l|}{$B$} \\
\hline small & 20 & $0.176 \pm 0.019$ & $1.833 \pm 0.311$ \\
\hline large & 100 & $0.173 \pm 0.011$ & $1.745 \pm 0.174$ \\
\hline \multicolumn{4}{|l|}{ C } \\
\hline small & 20 & $0.298 \pm 0.033$ & $3.775 \pm 0.533$ \\
\hline large & 100 & $0.299 \pm 0.015$ & $3.801 \pm 0.234$ \\
\hline \multicolumn{4}{|l|}{$D$} \\
\hline small & 20 & $0.117 \pm 0.013$ & $0.852 \pm 0.209$ \\
\hline large & 100 & $0.115 \pm 0.006$ & $0.839 \pm 0.094$ \\
\hline
\end{tabular}

* Values expressed as the means \pm standard deviations. 
Quality measures for the management of hydrocephalus

TABLE 2: Estimated numbers of years of observation required to obtain nonoverlapping $95 \%$ confidence intervals for the SARs of simulated practices*

\begin{tabular}{|c|c|c|c|c|c|c|c|c|c|}
\hline \multirow[b]{2}{*}{ Practice } & \multirow[b]{2}{*}{ Annual Patient Accrual (no.) } & \multicolumn{2}{|c|}{ Practice A } & \multicolumn{2}{|c|}{ Practice B } & \multicolumn{2}{|c|}{ Practice C } & \multicolumn{2}{|c|}{ Practice D } \\
\hline & & 20 & 100 & 20 & 100 & 20 & 100 & 20 & 100 \\
\hline \multirow[t]{2}{*}{$A$} & 20 & - & - & 48 & 57 & 1 & 1 & 2 & 1 \\
\hline & 100 & - & - & 49 & 64 & 1 & 1 & 1 & 1 \\
\hline \multirow[t]{2}{*}{ B } & 20 & - & - & - & - & 1 & 1 & 2 & 1 \\
\hline & 100 & - & - & - & - & 1 & 1 & 1 & 1 \\
\hline \multirow[t]{2}{*}{ C } & 20 & - & - & - & - & - & - & 1 & 1 \\
\hline & 100 & - & - & - & - & - & - & 1 & 1 \\
\hline \multirow[t]{2}{*}{ D } & 20 & - & - & - & - & - & - & - & - \\
\hline & 100 & - & - & - & - & - & - & - & - \\
\hline
\end{tabular}

largely by factors out of the control of the surgeon, namely the underlying brain disease responsible for the hydrocephalus. And a patient's perception of quality of life can be dissociated from functional capacity to a surprising degree. In a series of recent publications, Kulkarni and associates $^{9,10}$ developed and validated an instrument for measuring patient-perceived quality of life. Among the 51 elements of this Hydrocephalus Outcome Questionnaire (HOQ), only 1, "misses a lot of school due to illness," related in any way to the frequency of surgical complications. However, a subsequent analysis of quality of life, as measured by the HOQ among children followed up at the Hospital for Sick Children, demonstrated detrimental effects of the following clinical factors among others: numbers of shunt catheters, residential distance from the hospital, days hospitalized for shunt infection, and days hospitalized for shunt failure. Interestingly clinical factors accounted for only $23 \%$ of the measured variance in self-perceived quality of life in this study population. ${ }^{12}$ Avoidance of hospitalization for the treatment of complications seems therefore to be a meaningful good-although not the only one-for children with hydrocephalus and their families.

It is important for quality measures to be based on data that are accessible and auditable. In neurosurgical practices that utilize electronic billing records, the data required to calculate the SAR can be summoned with a few keystrokes on the basis of diagnostic and procedural codes. Likewise the RQ can be calculated from billing records, but it is particularly suitable for quality comparisons in web-based registries.

The simulated practices described here necessarily begin at Time 0 and grow to a steady state. In the real world pediatric neurosurgical practices seldom begin from nothing. Typically a new surgeon may join an existing practice, a practice may split, or two practices may merge. Such events can be expected to cause transient perturbations in quality metrics such as the SAR and RQ. Even though the growth of a practice from nothing is seldom observed in the real world, we described the growth phases of our simulations because they illustrate operational differences between the SAR and the RQ. The SAR approaches its steady-state value earlier in the growth phase of a developing practice than the RQ. This behavior suggests that it may be more stable than the RQ in the face of year-to-year fluctuations in new patient accrual that can occur on the basis of regional competition or through the addition or loss of personnel.

The simulations reported here generated standard deviations for the SAR and RQ that were small enough to allow detection of differences between Practices A, C, and D with grossly differing aggregate shunt survivals, but these metrics did not permit us to distinguish between Practices A and B. This failure may be regarded as a flaw insomuch as Practices A and B were different by definition. On the other hand, this failure may demonstrate that what we investigators judged to be an important theoretical difference in shunt survivals was in fact of little clini-

TABLE 3: Estimated numbers of years of observation required to obtain nonoverlapping $95 \%$ confidence intervals for the $R Q$ s of simulated practices

\begin{tabular}{|c|c|c|c|c|c|c|c|c|c|}
\hline \multirow[b]{2}{*}{ Practice } & \multirow[b]{2}{*}{ Annual Patient Accrual (no.) } & \multicolumn{2}{|c|}{ Practice A } & \multicolumn{2}{|c|}{ Practice B } & \multicolumn{2}{|c|}{ Practice C } & \multicolumn{2}{|c|}{ Practice D } \\
\hline & & 20 & 100 & 20 & 100 & 20 & 100 & 20 & 100 \\
\hline \multirow[t]{2}{*}{ A } & 20 & - & - & 38 & 72 & 1 & 1 & 2 & 1 \\
\hline & 100 & - & - & 45 & 179 & 1 & 1 & 1 & 1 \\
\hline \multirow[t]{2}{*}{ B } & 20 & - & - & - & - & 1 & 1 & 2 & 1 \\
\hline & 100 & - & - & - & - & 1 & 1 & 1 & 1 \\
\hline \multirow[t]{2}{*}{ C } & 20 & - & - & - & - & - & - & 1 & 1 \\
\hline & 100 & - & - & - & - & - & - & 1 & 1 \\
\hline \multirow[t]{2}{*}{ D } & 20 & - & - & - & - & - & - & - & - \\
\hline & 100 & - & - & - & - & - & - & - & - \\
\hline
\end{tabular}




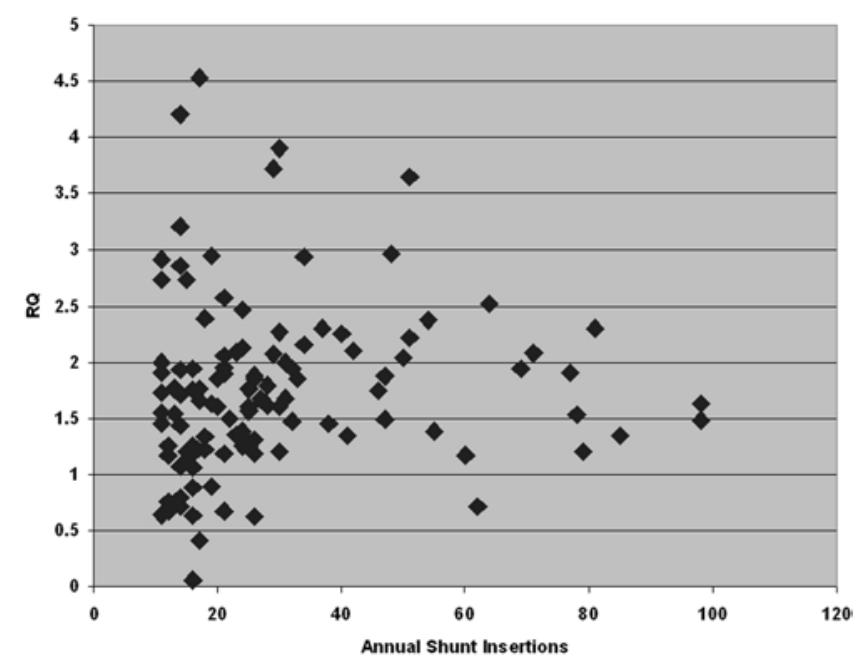

FIG. 3. Graph depicting RQs for CSF shunt surgery for the 117 hospitals that accounted for more than 10 admissions coded for initial shunt insertion in the $2009 \mathrm{KID}$.

cal significance. The calculation and use of the SAR and the RQ as quality measures must be evaluated prospectively in the field.

Implicit in the definition of the SAR is the expectation that all patients under care for hydrocephalus return to see their neurosurgeon at least annually. A policy of annual follow-up minimizes the SAR but does not affect the RQ. Among pediatric neurosurgical practices this policy is common, if not universal, but we are unaware of any data pertaining to its benefits. Adherence to such a policy is a strategy for quality improvement under the SAR metric. In theory a high degree of patient flux among different regional neurosurgical practices can vitiate assumptions underlying the concepts of the SAR and $\mathrm{RQ}$, but in our experience the bonds of a patient with hydrocephalus to a trusted neurosurgeon and to a familiar hospital are fairly stable.

The simulations described here were limited to CSF shunt surgery, but the SAR and RQ can be applied without modification to practices that perform ETV, as most pediatric neurosurgical practices probably do. Judicious selection of patients for treatment via ETV can be expected to minimize both the SAR and the RQ, and therefore it represents a strategy for quality improvement under both metrics.

Whether measurable quality differences exist among neurosurgical practices remains to be determined, but some population-based evidence has appeared in the literature. Cochrane and Kestle ${ }^{1}$ analyzed provincial health system databases in English-speaking Canada for the period from 1989 to 2001 for outcomes of CSF shunt surgery. They documented differences in overall failure rates and in infection rates in the aggregate between more and less experienced surgeons. These differences were highly statistically significant, but they were small. In a large-scale study utilizing data from the Pediatric Health Information System (PHIS) of the Child Health Corporation of America, Shah and associates ${ }^{18}$ reported better shunt survivals in the Northeast as compared with other regions of the US. In another study based on the PHIS,
S. E. Barton, J. W. Campbell, and J. H. Piatt Jr.

Simon and associates ${ }^{19}$ analyzed differences in CSF shunt infection rates among hospitals. Raw 24-month infection rates varied among hospitals from $2.5 \%$ to $12.3 \%$ per procedure, but in a multivariate analysis hospital and surgeon factors failed to retain significance. Methodological limitations of the PHIS for the study of CSF shunt surgery have been discussed..$^{13,18}$ Previous investigations have proceeded on the assumption that patient factors and technical surgical factors account for the observed variation in shunt outcomes, but indications for shunt revision can vary among practices as well. We are not aware of any published data pertinent to this possibility. Data in the current study demonstrate substantial variation in the RQ among busy pediatric centers. At some centers less than 1 revision was performed for every new shunt insertion; at others the ratio was almost 4:1. The causes for this variation are obscure, but they must be of great interest to surgeons, patients, and families. Whether this variation reflects differences in patient mix or true differences in the quality of care can be determined only by further research.

Our simulations and our calculations from the KID are merely illustrative, not normative. The simulations were based on old shunt survival data conveniently available to the senior author (J.H.P.). These data were derived from a mixed pediatric and adult institutional practice in the 1980s and may not reflect contemporary outcomes. ${ }^{14}$ The accuracy of procedural codes for shunt surgery in the KID is doubtful as well. There is no ICD-9-CM procedural code for externalization of the distal catheter of a shunt. Externalization of a shunt is not a definitive procedure falling within the definitions of SAR and RQ, but an unknown number of externalizations must have been counted in the current analysis under incorrect codes. Furthermore, roughly $10 \%$ of admissions that were coded for shunt insertion in the current analysis were actually coded for 2 or more shunt insertions. We counted these excess insertions as revisions. These considerations illustrate the imprecision with which codes may have been assigned in the original data collection process. The actual numerical values of the SAR and RQ developed in this report must not be construed as standards. As for any quality measure, the SAR and RQ can serve as benchmarks only on the basis of prospectively collected, auditable, and risk-adjusted data.

\section{Conclusions}

The SAR and RQ have attractive qualitative features as practice-based quality measures. They are conceptually accessible and reflect events of immediate concern to patients, families, and surgeons. Both metrics can be calculated readily from electronic billing data. And the $\mathrm{RQ}$, at least, exhibits clinically meaningful interhospital variation as applied to CSF shunt surgery. The SAR and RQ merit prospective field-testing as measures of quality in the management of childhood hydrocephalus.

\section{Disclosure}

The authors report no conflict of interest concerning the mate- 


\section{Quality measures for the management of hydrocephalus}

rials or methods used in this study or the findings specified in this paper.

Author contributions to the study and manuscript preparation include the following. Conception and design: Piatt, Campbell. Acquisition of data: Piatt, Barton. Analysis and interpretation of data: all authors. Drafting the article: Piatt. Critically revising the article: all authors. Reviewed submitted version of manuscript: all authors. Approved the final version of the manuscript on behalf of all authors: Piatt. Statistical analysis: Piatt. Administrative/technical/ material support: Piatt, Barton. Study supervision: Piatt.

\section{References}

1. Cochrane DD, Kestle JR: The influence of surgical operative experience on the duration of first ventriculoperitoneal shunt function and infection. Pediatr Neurosurg 38:295-301, 2003

2. Drake JM: Endoscopic third ventriculostomy in pediatric patients: the Canadian experience. Neurosurgery 60:881-886, 2007

3. Drake JM, Kestle JR, Milner R, Cinalli G, Boop F, Piatt J Jr, et al: Randomized trial of cerebrospinal fluid shunt valve design in pediatric hydrocephalus. Neurosurgery 43:294-305, 1998

4. Kestle J, Drake J, Milner R, Sainte-Rose C, Cinalli G, Boop F, et al: Long-term follow-up data from the Shunt Design Trial. Pediatr Neurosurg 33:230-236, 2000

5. Kestle JR, Drake JM, Cochrane DD, Milner R, Walker ML, Abbott R III, et al: Lack of benefit of endoscopic ventriculoperitoneal shunt insertion: a multicenter randomized trial. J Neurosurg 98:284-290, 2003

6. Kestle JR, Walker ML: A multicenter prospective cohort study of the Strata valve for the management of hydrocephalus in pediatric patients. J Neurosurg 102 (2 Suppl):141-145, 2005

7. Kulkarni AV: Quality of life in childhood hydrocephalus: a review. Childs Nerv Syst 26:737-743, 2010

8. Kulkarni AV, Drake JM, Mallucci CL, Sgouros S, Roth J, Constantini S: Endoscopic third ventriculostomy in the treatment of childhood hydrocephalus. J Pediatr 155:254-259.e1, 2009

9. Kulkarni AV, Drake JM, Rabin D, Dirks PB, Humphreys RP, Rutka JT: Measuring the health status of children with hydrocephalus by using a new outcome measure. J Neurosurg 101 (2 Suppl):141-146, 2004

10. Kulkarni AV, Rabin D, Drake JM: An instrument to measure the health status in children with hydrocephalus: the Hydrocephalus Outcome Questionnaire. J Neurosurg 101 (2 Suppl):134-140, 2004
11. Kulkarni AV, Riva-Cambrin J, Browd SR: Use of the ETV Success Score to explain the variation in reported endoscopic third ventriculostomy success rates among published case series of childhood hydrocephalus. Clinical article. J Neurosurg Pediatr 7:143-146, 2011

12. Kulkarni AV, Shams I: Quality of life in children with hydrocephalus: results from the Hospital for Sick Children, Toronto. J Neurosurg 107 (5 Suppl):358-364, 2007

13. Piatt JH Jr: A multicenter study of factors influencing cerebrospinal fluid shunt survival in infants and children. Neurosurgery 64:E1206, 2009 (Letter)

14. Piatt JH Jr, Carlson CV: A search for determinants of cerebrospinal fluid shunt survival: retrospective analysis of a 14-year institutional experience. Pediatr Neurosurg 19:233-242, 1993

15. Piatt JH Jr, Cosgriff M: Monte Carlo simulation of cerebrospinal fluid shunt failure and definition of instability among shunt-treated patients with hydrocephalus. J Neurosurg 107 (6 Suppl):474-478, 2007

16. Pollack IF, Albright AL, Adelson PD: A randomized, controlled study of a programmable shunt valve versus a conventional valve for patients with hydrocephalus. Neurosurgery 45:1399-1411, 1999

17. Sainte-Rose C, Piatt JH, Renier D, Pierre-Kahn A, Hirsch JF, Hoffman HJ, et al: Mechanical complications in shunts. Pediatr Neurosurg 17:2-9, 1991-1992

18. Shah SS, Hall M, Slonim AD, Hornig GW, Berry JG, Sharma V: A multicenter study of factors influencing cerebrospinal fluid shunt survival in infants and children. Neurosurgery 62:1095-1103, 2008

19. Simon TD, Hall M, Riva-Cambrin J, Albert JE, Jeffries HE, Lafleur B, et al: Infection rates following initial cerebrospinal fluid shunt placement across pediatric hospitals in the United States. Clinical article. J Neurosurg Pediatr 4:156-165, 2009

Manuscript submitted July 16, 2012.

Accepted January 2, 2013.

Please include this information when citing this paper: published online February 1, 2013; DOI: 10.3171/2013.1.PEDS12362.

Address correspondence to: Joseph H. Piatt Jr., M.D., Division of Neurosurgery, Alfred I. duPont Hospital for Children, 1600 Rockland Road, Wilmington, Delaware 19803. email: jpiatt@nemours. org. 